Scientific Paper

\title{
Influence of ultrasound on the clearance of toxic substances from Polysulfone hollow fiber membrane used in a dialyzer filter
}

\author{
Behrouz RASULI ${ }^{1}$, Naser TABKHI ${ }^{2}$, Ebrahim KALANTAR ${ }^{3}$, Vahid ZAREZADE ${ }^{4}$, Mohammad Bagher SHIRAN ${ }^{5, a}$ \\ ${ }^{I}$ Department of Radiology Technology, Behbahan Faculty of Medical Sciences, Behbahan, Iran \\ ${ }^{2}$ Samin Teb Soroush Company (Smart), Tehran, Iran \\ ${ }^{3}$ Department of Immunology, Faculty of Allied Medical Sciences, Iran University of Medical Sciences, Tehran, Iran \\ ${ }^{4}$ Department of Biochemistry, School of Medicine, Behbahan Faculty of Medical Sciences, Behbahan, Iran \\ ${ }^{5}$ Department of Medical Physics, Faculty of Medicine, Iran University of Medical Sciences, Tehran, Iran \\ ${ }^{a}$ E-mail address: shiranmb@yahoo.com
}

(received 2 February 2018; revised 22 June 2018; accepted 28 June 2018)

\begin{abstract}
The aim of this work was to investigate the potential of high frequency ultrasound for the cleaning of a Polysulfone (PSU) ultrafiltration membrane used in hemodialysis. The blood substitute solution flowing through the hollow fiber membrane used in the High Flux F60 dialyzers has been sonicated by ultrasonic (US) waves with 1 MHz frequency and $2 \mathrm{~W} / \mathrm{cm} 2$ in situ intensity .The solution was comprised of toxic compounds such as Urea, Creatinine, Vitamin B12 and Inulin. The effect of US was represented by comparison of the clearance in both OFF and ON ultrasound conditions. The results of these studies have been demonstrated that US can enhance the permeability of the PSU dialyzer membrane. Results revealed that US had significant effect ( $p$-value $<0.05$ ) on the clearance of Inulin as a large molecule $(5200 \mathrm{D})$ and accelerated its filtration by $28 \%$. The proposed dialysis method can be used alongside the existing dialysis machine.
\end{abstract}

Key words: hemodialysis; chronic kidney disease; ultrasound; hollow fiber membrane; filtration.

\section{Introduction}

The increasing incidence of non-communicable diseases, including diabetes and high blood pressure and complications of these diseases has made physicians and policy makers more aware of chronic kidney disease and renal replacement treatment. The purpose of dialysis is to remove the amount of toxic materials and water that has accumulated in the inter dialysis period by diffusion and ultrafiltration separation processes [1].

Long dialysis duration may challenge with daily programs as well as dialysis-related complications. The dialysis patients are mainly composed of elderly diabetic people with a heavy burden of comorbidities imposed by the disease. Therefore, reducing the time of dialysis procedure results in the reduction of these complications, bringing benefits to more patients and economic saving. It is noteworthy that dialysis adequacy should not be adversely affected by shortening of dialysis session duration.

The membrane is a decisive factor of the overall dialyzer function [2]. Membrane filtration rate reduction is one of the reasons for the long dialysis procedure. The major drawback of membrane filtration is fouling that can blind the dialyzer membrane and results in membrane performance deterioration during blood purification [3-5]. A similar problem exists in some industrial areas like wastewater treatment, desalination, food and dairy industries [6-9]. Many efforts have been made in recent years to dislodge and remove foulants from the membrane surface in order to enhance solute permeation using a variety of micro/ultrafiltration membranes. A number of studies have been performed applying external forces such as forward and backward flushing, air flushing, electrical and magnetic field and ultrasound to eliminate this problem [10$15]$.

Ultrasound are the mechanical vibration of the elastic medium particles around the equilibrium position with a frequency above $20 \mathrm{kHz}$. When ultrasound is propagating in a liquid or gaseous media, a local thinning and thickening of vibrating particles of a medium occurs causing changes in its density and pressure. High intensity and relatively low frequency focused ultrasound can induce thermal and nonthermal effects in elastic media. Non-thermal effects of ultrasound appear in the form of such phenomena as cavitation, acoustic micro-streaming or strains on cell membranes. Range of biological effects induced in tissue by ultrasound depends on the acoustic parameters of the ultrasound beam used, such as local intensity, frequency, pulse duration, duty-factor and exposure time, as well as on acoustic and thermal properties of media of propagation such as density, sound speed, viscosity, 
thermal conductivity. Each of the above-mentioned physical parameters can in some way affect the flow of blood through the membrane. These phenomena can also prevent clogging of the membrane pores during filtration by increasing stress / strain forces and shock waves associated with cavitation [16].

There has been a significant amount of research investigating on cleaning of industrial membrane in the presence of ultrasound $[15,17,18]$. There are a few studies conducted on the membranes used in medical equipment like dialysis filter $[4,19]$. Therefore, the aim of this work was to examine the potential of ultrasound with a $1 \mathrm{MHz}$ frequency and $2 \mathrm{~W} / \mathrm{cm}^{2}$ local intensity for cleaning the hollow fiber ultrafiltration membrane made of polysulfone (PSU) and used in a commercially available high flux dialyzer. Current study compares clearance of the blood substitute including Urea, Creatinine, vitamin B12 and Inulin in presence and absence of ultrasound used.

\section{Materials and Methods}

Experiments were performed using High-Flux dialyzer (supplied by Soha 1, Iran and was produced by Fresenius Medical Care, Germany) in five phases. The dialyzer contained hollow fiber synthetic Polysulfone membranes. Table 1 represents the characteristics of the dialyzer used in this study.

Ultrasound waves were generated by three piezoelectric flat transducers operating at a frequency of $1 \mathrm{MHz}$ and maximum intensity output of $2 \mathrm{~W} / \mathrm{cm}^{2}$ (measured in the near field of ultrasound field) was designed in the department of Medical Physics at Iran University of Medical Sciences (IUMS). Each transducer has an area of $4 \mathrm{~cm}^{2}$ and generated pulses with a duration of $0.3 \mathrm{~s}$ and a repetition period of $0.5 \mathrm{~s}$. The transducers were fixed on a Plexiglas frame (Figure 1). All experiments were run at a constant frequency of $1 \mathrm{MHz}$ and intensity of $2 \mathrm{~W} / \mathrm{cm}^{2}$. All efforts have been made in order to cavitation-free conditions be adopted by using a high ultrasound frequency $(1 \mathrm{MHz})$ to decrease the probability of cavitation (in comparison to lower frequencies that cavitation is dominant) and a bubble trapper was used in our experiments to remove any bubbles that may produce during the ultrasound irradiation.

\section{Penetration capability of ultrasound into the dialyzer}

The shape and the absorption coefficient of the dialyzer housing material may affect the sound penetration into the dialyzer housing. Each transducer producing the ultrasonic field was calibrated separately using radiation force method. The filter or dialyzer housing was made of transparent polycarbonate elements connected by a silicone rubber O-ring and a polyurethane adhesive [20]. Membrane's fibers of the dialyzer were removed from its housing and replaced by a cylindrical-shaped thick paper. An aqueous solution of Methylene blue dye with $10 \mathrm{mg} / \mathrm{l}$ concentration was prepared. It was used as an ultrasound water bath and necessary to record
Table 1. High-flux dialyzer technical characteristics.

\begin{tabular}{lc}
\hline \hline Dialyzer type & High Flux F60 - Microfiltration \\
Membrane type & Hollow fiber \\
Membrane material & Synthetic Polysulfone \\
Dialyzer hosing material & Polycarbonate \\
Fiber inner diameter $(\mu \mathrm{m})$ & 200 \\
Fiber wall thickness $(\mu \mathrm{m})$ & 40 \\
MWCO $(\mathrm{kDa})$ & 30 \\
Pore size $(\mu \mathrm{m})$ & 0.01 \\
$\begin{array}{l}\text { Ultrafiltration coefficient } \\
(\mathrm{ml} / \mathrm{h} . \mathrm{mm} H \mathrm{H})\end{array}$ & 40 \\
$\begin{array}{l}\text { Effective surface membrane inside } \\
\text { a dialyzer }\left(\mathrm{m}^{2}\right)\end{array}$ & 1.3 \\
Blood flow range $(\mathrm{ml} / \mathrm{min})$ & $200-300$ \\
\hline \hline
\end{tabular}

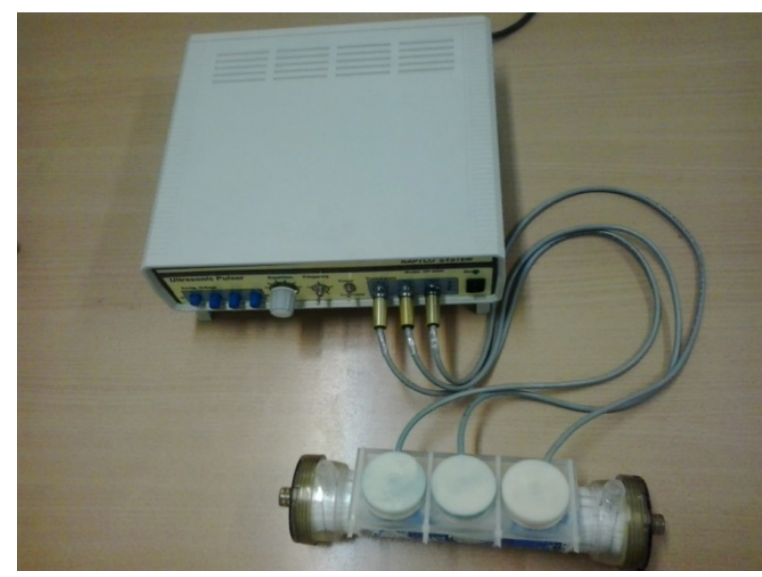

Figure 1. Photo of three transducers mounted on a Plexiglas plate and placed at a distance of $\mathbf{5} \mathbf{~ m m}$ from the dialyzer housing.

the ultrasonic field pattern, if it existed. Afterwards, the paperfilled housing was immersed in the prepared solution for depicting the ultrasound pattern on the cardboard paper inside the housing. All chemicals throughout the experiments were supplied by Merck Inc. Continuous ultrasound waves at $1 \mathrm{MHz}$ frequency and intensity of $2 \mathrm{~W} / \mathrm{cm}^{2}$ was applied to the paperfilled housing for 5 minutes at $1 \mathrm{~cm}$ distance between the transducers and dialyzer housing.

\section{Temperature monitoring}

Temperature control is crucial to prevent a potential damage to the blood cells at the next stage of this research. During the experiments, a constant water temperature of $37^{\circ} \mathrm{C}$ in the water bath was maintained by means of a thermostatic system. Water was used as a coupling medium between transducers and the filter. The temperature inside the dialyzer filter at the center of ultrasound field was measured by a NX7 digital thermometer (Hanyoung, South Korea) with a range of $0-50{ }^{\circ} \mathrm{C}$ and an accuracy of $\pm 0.5 \%$.

\section{Preparing the blood substitute}

Blood substitute was prepared based on the Fresenius quality control procedure [21]. This solution was comprised of toxic compounds such as Urea, Creatinine, Vitamin B12 and Inulin 
at concentrations similar to those found in the patient's blood before starting the hemodialysis treatment. To develop the blood substitute, Urea, Creatinine, Vitamin B12 and Inulin were dissolved in 24 liters of double distilled water to attain concentrations of $1 \mathrm{~g} / \mathrm{l}, \quad 0.15 \mathrm{~g} / \mathrm{l}, \quad 0.05 \mathrm{~g} / \mathrm{l}, \quad$ and $0.2 \mathrm{~g} / \mathrm{l}$, respectively. Urea and Creatinine (60 and 113 Daltons, respectively) are relatively small molecules. Vitamin B12 (1355 Daltons) was used as a marker for middle molecules and Inulin is a large molecule (5200 Daltons). The blood substitute prepared in this way was flowing into the High-Flux dialyzer.

\section{Ultrasonic cleaning}

Three transducers were mounted on a pre-designed and built Plexiglas holder. The dialyzer was placed at an axial distance of $5 \mathrm{~mm}$ from the radiating surface of the ultrasonic transducers as illustrated in Figure 1. To provide a coupling medium for penetrating of ultrasound waves into the dialyzer, the system was placed in a glass tank $(40 \mathrm{~cm} \times 25 \mathrm{~cm} \times 30 \mathrm{~cm})$ as an ultrasonic bath filled with distilled water. The experimental setup is shown schematically in Figure 2.

Dialysis set (supplied by Soha 1, Iran) including arterial and venous transparent PVC tubes were used to connect the corresponding ports of the immersed dialyzer to the blood substitute and dialysate feed tank (Haake W26 bath, Thermo Electron Corp, USA). The feed tanks were controlled with temperature control system and an immersion circulator (DC3 \& DC5 Haake fisons, USA) so that the liquids were kept at a constant temperature during the experiments. Both the feed baths were made of stainless steel with 241 capacity and $34 \mathrm{~cm}$ $\times 56 \mathrm{~cm} \times 40 \mathrm{~cm}$ dimensions. The dialyzer was fed by an EasyLoad peristaltic pump heads (Masterflex I/P, USA) at a constant pressure of $1 \mathrm{~atm}$. The flow rate through the blood substitute circuit was held constant at $300 \mathrm{ml} / \mathrm{min}$, while the flow rate through the dialysate circuit was maintained in the level of $500 \mathrm{ml} / \mathrm{min}$. The solution temperature was kept at $37^{\circ} \mathrm{C}$ according to the physiological temperature of a body. The priming and soaking were conducted by passing distilled water through the dialyzer for two minutes before starting main experiments.

\section{Sonication of Dialyzer \\ Filtration without sonication}

A sample was taken before starting the circulation (sample $\mathrm{A}_{0}$ ). Then, the blood substitute was circulated through the HighFlux dialyzer and samples were taken every five minutes for 60 minutes (samples $\mathrm{A}_{1}-\mathrm{A}_{12}$ ). No dialyzer sonication was occurred in this part. All samples were kept at $37^{\circ} \mathrm{C}$ in an incubator (Inculab, Shimadzu, Japan) until analyzed.

\section{Filtration with sonication}

The above procedure was repeated, but this time in the presence of ultrasound. A sample was taken before starting the circulation (sample $\mathrm{B}_{0}$ ). Then, the blood substitute was circulated through the dialyzer. To measure clearance, "Blood out" concentrations were sampled periodically every five minutes for 60 minutes (samples $\mathrm{B}_{1}-\mathrm{B}_{12}$ ).

The effect of sonication on dialyzer cleaning was presented by comparing the clearance for both conditions: in the presence of ultrasound waves $(\mathrm{ON})$ and in their absence (OFF). Sample analysis was performed using a Genesis 10 UV spectrophotometer (Thermo scientific, USA). The following equation was used to calculate the clearance.

$C=Q_{\text {Bin }}\left(1-\frac{C_{\text {out }}}{C_{\text {in }}}\right)$

Eq. 1

Where $\mathrm{C}$ is clearance, $\mathrm{Q}_{\mathrm{Bin}}$ blood substitute input flow rate, $\mathrm{C}_{\text {out }}$ output concentration and $\mathrm{C}_{\mathrm{in}}$ input concentration of each material in the samples taken. Each experiment was repeated at least three times and acquired raw data were analyzed statistically using paired t-test and SPSS v16.

\section{Results}

Figures 3-5 show the ultrasound pattern recorded on the paper inside the dialyzers housing. The intensity of ultrasound, which during the penetration of the dialyzer did not induce a significant increase in temperature inside it, was considered safe.

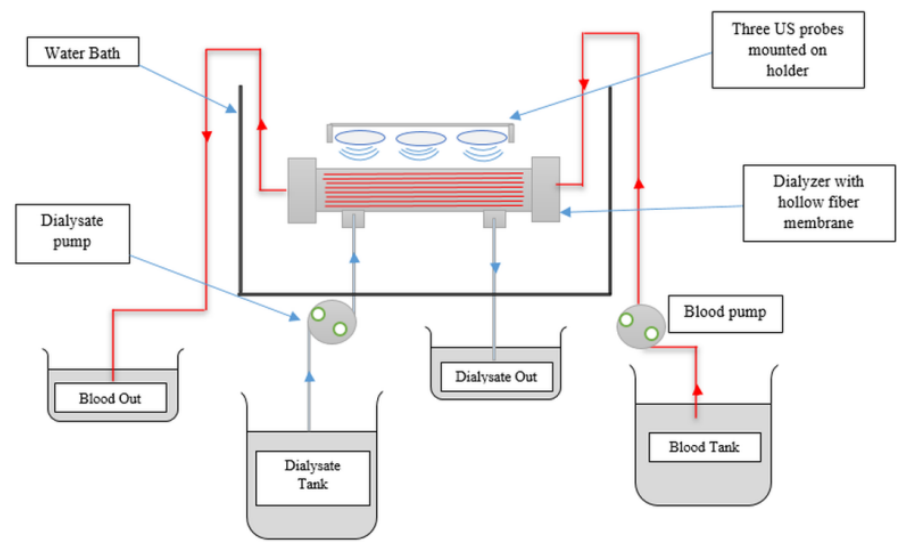

Figure 2. A block diagram used for a filtration experiment and containing an ultrasonic module placed close to the dialyzer.
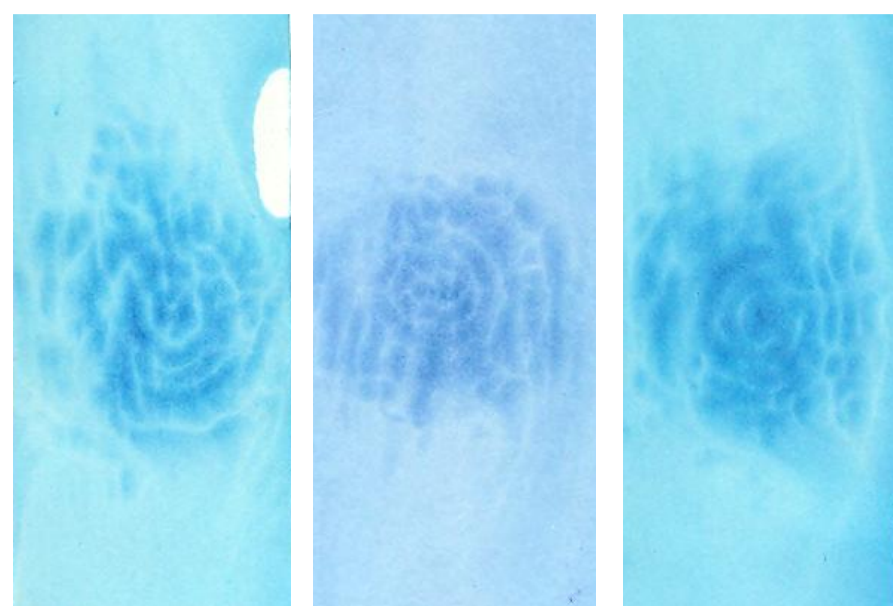

Figures 3-5. Images of radial cross-sections of ultrasonic fields generated by 3 transducers $\left(1 \mathrm{MHz}, 2 \mathrm{~W} / \mathrm{cm}^{2}\right)$ and obtained on paper, placed at a distance of $1 \mathrm{~cm}$ from them, after 5 minutes of sonication. 
Figure 6 shows the temperature variations in the presence of ultrasound when distilled water was flowing with constant flow rate of $300 \mathrm{ml} / \mathrm{min}$. Temperature measurement was performed in a point corresponding to the center of the acoustic field obtained in the previous part, in order to measure the maximum heat released. During 3.5 hours of sonication of the filter, the temperature at the center of the filter was raised by $2.2^{\circ} \mathrm{C}$.

The experiments with the blood substitute solution were performed in presence and absence of ultrasound. Samples of $30 \mathrm{ml}$ were collected from the blood substitute circuit output every five minutes. Figures 7-10 illustrate the main findings of this study that compare clearance of Urea, Creatinine, B12 and Inulin with and without exposure to ultrasound. Statistical results are presented in Table 2 , including average, SD and $\mathrm{P}$ -

Table 2. Statistical parameters of clearance $(\mathrm{ml} / \mathrm{min})$ obtained for materials tested in the presence and without US.

\begin{tabular}{llllll}
\hline \hline & & B $_{12}$ & Creatinine & Urea & Inulin \\
\hline \multirow{2}{*}{ Average } & with US & 116.75 & 162.75 & 176.48 & $\mathbf{8 3 . 7 6}$ \\
& without US & 114.65 & 168.53 & 170.74 & $\mathbf{6 5 . 5 2}$ \\
\multirow{2}{*}{ SD } & with US & 1.08 & 2.60 & 3.36 & 5.29 \\
& without US & 7.28 & 2.80 & 13.60 & 9.11 \\
\hline p-value & & $>0.05$ & $<\mathbf{0 . 0 5}$ & $>0.05$ & $<\mathbf{0 . 0 5}$ \\
\hline \hline
\end{tabular}

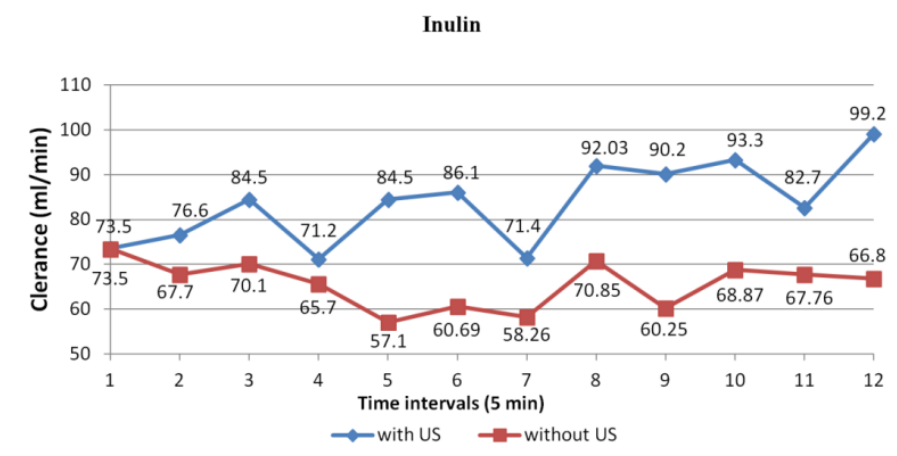

Figure 7. Clearance of Inulin in the presence and without ultrasound.

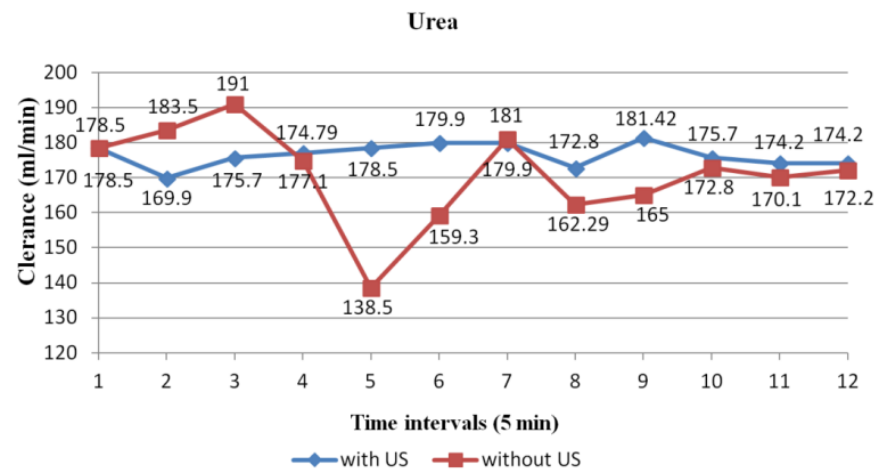

Figure 9. Clearance of Urea in the presence and without ultrasound. values. Significant changes were confirmed for Inulin clearance ( $p$-value $<0.05)$. Also, the Creatinine clearance displayed a negative correlation with ultrasound.

\section{Discussion}

A dramatic increase in the number of end-stage renal disease patients has made physicians and policy makers more aware of chronic kidney disease and dialysis therapy. In the current study, experiments were performed with a hollow fibercontained membrane dialysis system coupled with three ultrasonic transducers to investigate the role of ultrasonic in the clearance recovery of toxic materials.

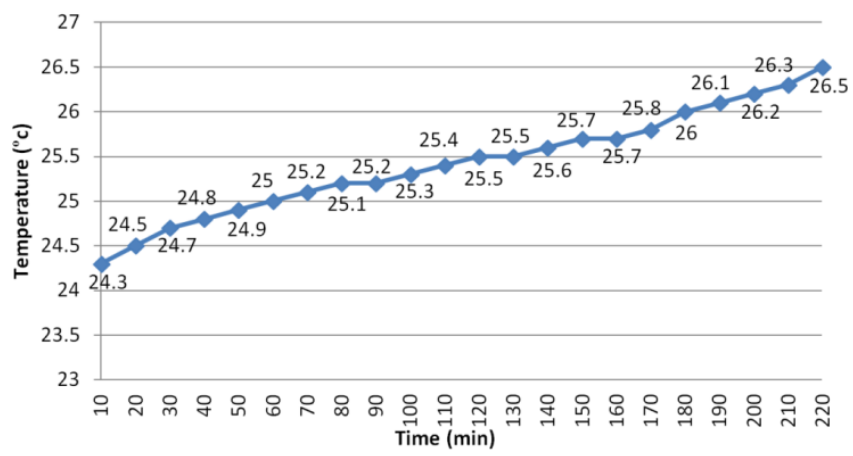

Figure 6. Temperature variations inside the dialyzer in the presence of US.

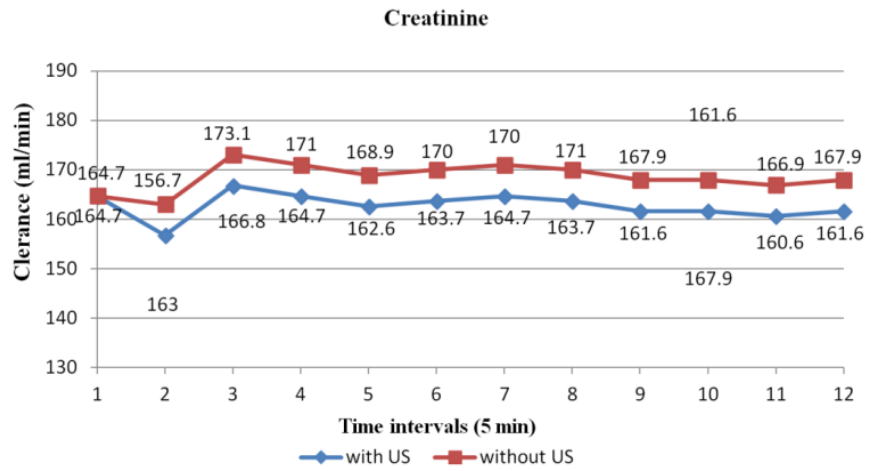

Figure 8. Clearance of Creatinine in the presence and without ultrasound.

B12

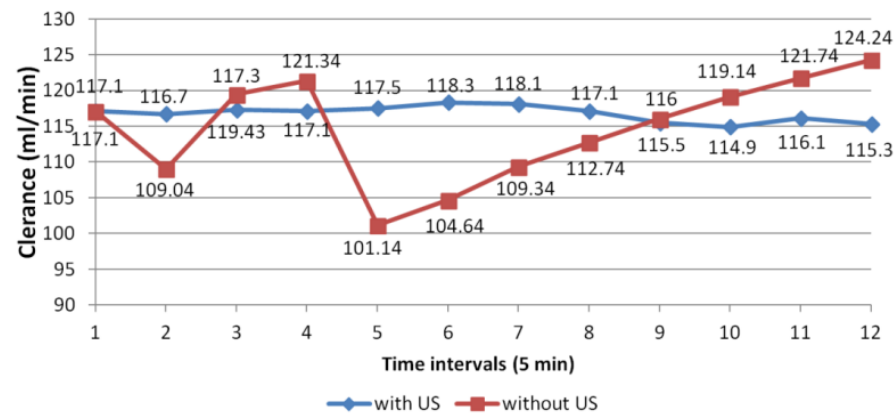

Figure 10. Clearance of $B 12$ in the presence and without ultrasound. 
Figures 3-5 show the penetration ability of ultrasound waves across the dialyzer filter. The radial cross-sections of the ultrasonic field, recorded for the central plane of the dialyzer are clearly visible and are very similar to the field pattern reproduced on the paper by Sarvazian method [22]. The recorded patterns ensured us that the absorption, scattering and reflection did not have much effect on propagation of sound in the filter.

Figure 6 shows temperature increase during 3.5 hours of sonication of the filter with distilled water flowing through the blood flow path, the maximum temperature at the center of ultrasonic field was raised by $2.2^{\circ} \mathrm{C}$. In a real dialysis treatment, the dialysate is also flowing with a flow rate of 500 $\mathrm{ml} / \mathrm{min}$ that causes conduction and convection effects and adjusts the blood temperature. This means that the increase in the blood temperature in a real hemodialysis process is less than our finding. This is indicating that ultrasound waves most probably have no adverse effect on the blood substructures. However, hemodialysis normally increases body temperature that should be controlled. The use of low temperature dialysate can lower the body temperature as well as increase cardiac contractility [23].

The high flux F60 membrane used in this study has the average pore diameter of $0.01 \mu \mathrm{m}$ and a MWCO (molecularweight cutoff) of $30 \mathrm{kD}$. The MWCO typically refers to the smallest average molecular mass of a standard molecule that will not effectively diffuse across the dialysis membrane. There is no direct correlation or conversion between a 2-dimensional metric lengths $(\mu \mathrm{m})$ and a 3-dimensional molecular size $(\mathrm{kD})$ but pore sizes typically range from $1-10 \mathrm{~nm}$ for $1-50 \mathrm{kD}$ MWCO membranes. What this means is that the toxic materials, including Urea $(60 \mathrm{D})$, Creatinine $(113 \mathrm{D})$ and vitamin B12 (1355 D) that their molecular weights are much smaller than the nominal MWCO, can easily pass through the membrane due to diffusion without the need for ultrasoundenhanced membrane filtration in cross-flow. In other words, sonication with ultrasound has not a positive effect on the clearance of tested particles, as expected, but may prevent their attachment to other molecules. The obtained experimental results indicate that ultrasound waves have no significant effects on the clearance of Creatinine.

Figure 7 shows the effect of ultrasound waves on filtration of hollow fiber dialysis membranes. With respect to the Fresenius Polysulfone high flux dialyzer characteristics, highflux membrane has been shown to remove a large portion of middle-weight molecules (500-2000 D) and has not a sieving coefficient of $100 \%$ for large molecules such as Inulin [21]. Therefore, Inulin can accumulate near the inner surface of the membrane a concentrated boundary layer, known as a "cake layer", can potentially clog the pores and affect the dialyzer performance, which will worsen the treatment.

Statistical analysis ( $p$-value $<0.05)$ indicated that the presence of ultrasound during dialysis in a blood equivalent solution has a significant effect on Inulin clearance. The results showed that in the presence of ultrasound, the Inulin clearance increased by $28 \%$. This research demonstrated that ultrasound enhanced the permeability of the dialysis membrane due to the effect of their radiation force, mechanical movement of the dialysis fiber and acoustic streaming of the blood.

In this study, it has been attempted to approach cavitationfree condition by selecting high frequency and low intensity ultrasound [24]. Cavitation, which leads to an increase in oscillation and transient collapse of small bubbles of gas, generating shock waves, and has the potential to cause membrane damage [15]. Liquid media subjected to ultrasound at high frequency ( $\mathrm{f}>1 \mathrm{MHz}$ ) are particularly susceptible to the occurrence of the acoustic streaming, inducing them to vortex motions ensuring the mixing of such medium, which plays a significant role in membrane cleaning [25].

At frequencies higher than $1 \mathrm{MHz}$, cavitation bubble collapse occurs to a lesser extent than at low frequencies, and therefore, damage to the membrane is less likely $[10,15]$. The acoustic streaming increases with the increase in frequency of ultrasound waves and their intensity. As a result of increased movement of fluid due to the spread of micro-streaming, its permeability increases [24]. Ultrasound-enhanced membrane filtration has not yet been widely commercialized. The key obstacles to the commercialization of this innovative technology are the insufficient level of technology for the production of stable transducers for membrane filtration and the need to control membrane erosion, particularly when dealing with patient's life [26].

To our knowledge, no comprehensive studies have not yet been conducted to evaluate the effect of ultrasound on the filtration of the hollow fiber dialysis membranes for cases when blood or a blood substitute flows inside the dialyzer. The first researchers who investigated the application of ultrasound to accelerate the electrolyte diffusion of electrolyte through a cellophane dialysis membrane were Li et al. They found that permeability increases with sonication time. They also stated that, the lifetime of a membrane decreases with increasing ultrasound intensity [27]. However, application of ultrasound frequently appears in many studies and recently their applications have gained extensive importance. All these studies demonstrated that ultrasound have a potential to enhance filtration of industrial membranes [15,25].

Shiran et al evaluated clearance of a blood equivalent solution and human whole blood flowing through a high flux dialyzer. Their findings revealed the potential capability of $1 \mathrm{MHz}$ ultrasound with intensity of $1 \mathrm{~W} / \mathrm{cm}^{2}$ to increase the clearance of Urea and Inulin [19]. There is a compliance between our findings with the results reported in the abovementioned study. Likewise, the Inulin clearance analysis yielded good correspondence between our findings with the manufacturer's claims. Many studies showed that ultrasound has the potential to cause membrane damage, therefore ultrasound have to be used with care [15]. This work is in progress and further analysis will be reported subsequently. Further research, including assessment of pulsed ultrasound, more transducers, varied frequencies and intensities, more 
realistic blood substitute and other dialyzers available on the market will be performed.

\section{Conclusion}

The dialysis acceleration device proposed in this study could reduce the time necessary for dialysis and potentially save thousands of man hours for physicians, nurses, technicians, and patients, with enormous economic profit. It may be beneficial to the development of such an innovative ultrasound technology. This technology can be used as a complementary to existing dialysis equipment. The ultimate goals of such systems are to reduce the duration of the hemodialysis sessions leading to an increased availability of dialysis in patients with end-stage renal disease (ESRD) as well as to improve or at least maintain the adequacy of hemodialysis.

\section{Acknowledgement}

The authors would like to thank the Helal Iran Medical Device Company (SOHA 1) especially Quality Control Section staffs for sharing their important information with us during this research.

\section{Funding}

This work was supported by Iran University of medical Sciences (IUMS) (grant number 848).

\section{References}

[1] Himmelfarb J, Ikizler TA. Hemodialysis. N Eng J Med. 2010;363(19):1833-1845.

[2] Mineshima M. The past, present and future of the dialyzer. Contrib Nephrol. 2015;185:8-14.

[3] Röckel A, Hertel J, Fiegel P, et al. Permeability and secondary membrane formation of a high flux polysulfone hemofilter. Kidney Int. 1986;30(3):429-432.

[4] Nii S, Oketani S, Kawaizumi F, Takahashi K. Effects of Ultrasonic Irradiation on Solute Permeation through a Dialysis Membrane. J Chem Eng Japan. 2005;38(7):497-501.

[5] Li H, Ohdaira E, Ide M. Enhancement in Diffusion of Electrolyte through Membrane Using Ultrasonic Dialysis Equipment with Plane Membrane. Jap J Appl Phys. 1995;34(5S):2725.

[6] Cai M, Li W, Liang H. Effects of ultrasound parameters on ultrasound-assisted ultrafiltration using cross-flow hollow fiber membrane for Radix astragalus extracts. ChemEng Proc: Process Intensification. 2014;86:30-35.

[7] Lamminen MO, Walker HW, Weavers LK. Cleaning of particle-fouled membranes during cross-flow filtration using an embedded ultrasonic transducer system. J Membr Sci. 2006;283(1-2):225-232.

[8] Li J, Hallbauer DK, Sanderson RD. Direct monitoring of membrane fouling and cleaning during ultrafiltration using a non-invasive ultrasonic technique. J Membr Sci. 2003;215(1-2):33-52.

[9] Maskooki A, Kobayashi T, Mortazavi SA, Maskooki A. Effect of low frequencies and mixed wave of ultrasound and EDTA on flux recovery and cleaning of microfiltration membranes. Sep Purif Technol. 2008;59(1):67-73.

[10] Masselin I, Chasseray X, Durand-Bourlier L, et al. Effect of sonication on polymeric membranes. J Membr Sci. 2001;181(2):213220.

[11] Jin W, Guo W, LÜ X, et al. Effect of the Ultrasound Generated by Flat Plate Transducer Cleaning on Polluted Polyvinylidenefluoride Hollow Fiber Ultrafiltration Membrane. Chin J Chem Eng. 2008;16(5):801-804.

[12] Wang X-1, Li X-f, Fu X-q, et al. Effect of ultrasound irradiation on polymeric microfiltration membranes. Desalination. 2005;175(2):187-196.

[13] Kyllönen H, Pirkonen P, Nyström M, et al. Experimental aspects of ultrasonically enhanced cross-flow membrane filtration of industrial wastewater. Ultrason Sonochem. 2006;13(4):295-302.

[14] Cai M, Zhao S, Liang H. Mechanisms for the enhancement of ultrafiltration and membrane cleaning by different ultrasonic frequencies. Desalination. 2010;263(1-3):133-138.

[15] Kyllönen HM, Pirkonen P, Nyström M. Membrane filtration enhanced by ultrasound: a review. Desalination. 2005;181(1-3):319335.

[16] Li X, Yu J, Nnanna AGA. Fouling mitigation for hollow-fiber UF membrane by sonication. Desalination. 2011;281:23-29.

[17] Chai X, Kobayashi T, Fujii N. Ultrasound-associated cleaning of polymeric membranes for water treatment. Sep Purif Technol. 1999;15(2):139-46.

[18] Chai X, Kobayashi T, Fujii N. Ultrasound effect on cross-flow filtration of polyacrylonitrile ultrafiltration membranes. J Membr Sci. 1998;148(1):129-135.

[19] Shiran MB, Barzegar Marvasti M, Shakeri-Zadeh A, et al. Enhancement of Toxic Substances Clearance from Blood Equvalent Solution and Human Whole Blood through High Flux Dialyzer by 1 MHz Ultrasound. J Biomed Phys Eng. 2017;7(2):107-116.

[20] Care FM. Dialysers and Filters Product Range. In: Care FM, editor. Deutschland GmbH 2007. 
[21] Care FM. Fresenius Polysulfone High-Flux Dialyzers. In: Care FM, editor. Deutschland GmbH

[22] Watmough DJ, Quan KM, Shiran M, editors. The Sarvazyan method of mapping ultrasound fields. IEE Colloquium on Ultrasound Instrumentation; 199010 May 1990.

[23] van der Sande FM, Wystrychowski G, Kooman JP, et al. Control of Core Temperature and Blood Pressure Stability during Hemodialysis. Clin J Am Soc Nephrol. 2009;4(1):93-98.

[24] Hesson JR. Fundamentls of Ultrasound Cleaning. United States: Hessonic Ultrasounic.

[25] Kobayashi T, Kobayashi T, Hosaka Y, Fujii N. Ultrasound-enhanced membrane-cleaning processes applied water treatments: influence of sonic frequency on filtration treatments. Ultrasonics. 2003;41(3):185-190.

[26] Parker PM. Hemodialysis, A Medical Dictionary, Bibliography and Annotated Research Guide to Internet References. ICON Health Publications; 2004. 408 p.

[27] Li H,, Ohdaira E, Ide M. Effect of Ultrasonic Irradiation on Permeability of Dialysis Membrane. Jap J Appl Phys. 1996;35(5S):3255. 